\title{
CT Evaluation of Malignant PNS Mass and Histopathological Correlation
}

\author{
Tahmina Islam ${ }^{1}$, Salauddin Al-Azad ${ }^{2}$ Lubna Khondker $^{3}$, Sabina Akhter ${ }^{4}$ \\ ${ }^{1}$ Radiologist, Dept of Radiology \& Imaging, Dhaka Medical College Hospital, Dhaka; ${ }^{2}$ Professor, Department of Radiology \& Imaging, BSMMU, \\ Dhaka; ${ }^{3}$ Assistant Professor, Dept of Dermatology and venereology, BSMMU, Dhaka; ${ }^{4}$ Radiologist, Dept of Radiology \& Imaging, NIDCH, Dhaka.
}

\begin{abstract}
:
Background: Computed tomography (CT) is the gold standard for exact delineation of paranasal sinus(PNS) disease. There are many radiologically important diseases of paranasal sinuses. Objective: to evaluate the malignant PNS mass by computed tomographic image and the findings of this modality were compared with histopathological result. Methods: It was a cross sectional type of study and carried out with suspected PNS mass having patients during January 2009 to October 2010. Results: The mean age of the patients was $35.95 \pm 18.24$ and common complaints of the patients were nasal obstruction (73.7\%) and maximum 53.9\% patients had PNS mass in maxillary sinuses. Out of 76 cases $21.1 \%$ found malignant mass on CT and after histopathology $19.7 \%$ had malignant mass. Out of all cases 14 were diagnosed as malignant PNS mass by CT scan and confirmed by histopathological evaluation and they were true positive. Two cases were diagnosed as malignant PNS mass by CT scan but not confirmed by histopathological findings and they were false positive. Of 60 cases, which were diagnosed by CT scan, one was confirmed as malignant and 59 were benign by histopathology. They were false negative and true negative respectively. Sensitivity of CT scan to diagnose malignant PNS mass was $93.3 \%$, specificity $96.7 \%$, positive predictive value $87.5 \%$, negative predictive value $98.3 \%$ and accuracy $96.1 \%$. Conclusion: CT scan of the malignant para nasal sinus mass provides more information and better image quality and CT diagnosis correlate well with the findings of histopathology.
\end{abstract}

Key words: CT of PNS mass, histopathology of PNS mass.

\section{Introduction:}

There are many radiologically important diseases of paranasal sinuses. Acute sinusitis, nasal polyposis, mucoceles, different types of fungal diseases, Wegener's granulomatosis and different types of benign to life threatening malignant tumours are important. ${ }^{1}$ The diagnosis of sinus neoplasia remains a clinical problem for most physicians treating large numbers of patients with sinus complaints. Unfortunately, the signs and symptoms of sinus neoplasia are largely those of sinus inflammatory disease and delay in diagnosis is common. Sinus neoplasia is fortunately an uncommon tumor, representing $<4 \%$ of neoplasms of the head and neck. ${ }^{2}$ CT (computed tomography) play complementary roles in the evaluation of sinus neoplasia. CT continues to play a key role in the evaluation of the bony changes accompanying the tumor

Address for Correspondence: : Dr Tahmina Islam, Radiologist, Dept of Radiology \& Imaging, DMCH Dhaka.

Mobile: 01612132170
[BSMMU J 2013; 6 (1) : 33-37]

and in helping to determine the surgical approach to the tumor in paranasan sinus region. ${ }^{3}$ The radiologic examination is considered sometimes a complementary or supplementary diagnostic procedure to the clinical findings. ${ }^{4}$ Considerable progress in paranasal sinus imaging has occurred during the past decade as a result of refinements in CT. CT scanning has the ability to obtain thin-slice, high-resolution sectional images. CT has a higher intrinsic image contrast, whereas conventional radiography has very limited image contrast. CT scanning provides three-dimensional information, whereas conventional radiography produces two-dimensional images. For these reasons, CT scanning has largely supplanted conventional radiography for evaluation of the paranasal sinuses. $^{5}$ CT scan is an imaging modality of choice for evaluation of a suspected PNS mass. It provides useful information in regards to: location of the mass, characteristics of the mass (fat, fluid, solid); likely origin, mode of extension or spread. ${ }^{6}$ Recent advances in imaging techniques have made it possible to detect various PNS 
mass with the aid of computed tomography (CT) and magnetic resonance (MR) imaging. ${ }^{7}$ Radiology and Imaging department of Bangabandhu Sheikh Mujib Medical University is a promising centre for the diagnosis of different type of diseases. Every day a huge number of patients attend this department for conventional X-ray and CT scan. Considerable number of these patients have come from otolaryngology outpatient and in patients provisionally diagnosed PNS mass. Present study was designed to evaluate the malignant PNS mass by $\mathrm{CT}$ (computed tomographic image) and findings of this modality were compared with histopathological result.

\section{Methods:}

A cross sectional study was carried out in the department of Radiology \& Imaging, Bangabandhu Sheikh Mujib Medical University and Dhaka Medical College Hospital, Dhaka in collaboration with department of Otolaryngology and Department of Pathology of the same hospital. This study was carried out from January 2009 to October 2010 and samples were selected purposively among the patients. Inclusion criterias were -patients with PNS mass evaluated clinically and patients with PNS mass evaluated by PNS radiograph. Exclusion criterias were patients refusing to do CT scan and patients who were unwilling to do histopathology. A pre-tested questionnaire were used as research instruments. Statistical analysis of the results were presented in tables and figures. For the validity of study outcome, sensitivity, specificity, accuracy, positive predictive value and negative predictive value of CT scan in the diagnosis of PNS mass was calculated after confirmation of the diagnosis by histopathology.

\section{Scanning technique of CT scan and Histopathological correlation}

All patients were undergone CT examination of PNS at the department of Radiology and Imaging, BSMMU, Dhaka. All CT scan was performed with a third generation CT, Hitachi W2000 (3-5) mm thick contiguous slice was taken. These scans were obtained using $120 \mathrm{kv}, 150 \mathrm{~mA}$, $7.5 \mathrm{~mm}$ collimation and $1 \mathrm{sec}$ scanning time for 1 slice. Both pre and post contrast scan was performed. $50 \mathrm{ml}$ of non ionic water soluble iodinated contrast medium of 370 strength was administered. Immediately after completion of bolus injection 3-5 mm contiguous slice was obtained. The postoperative resected tissues were examined histopathologically in the respective department and then the collected reports were correlated with findings of CT.

\section{Result :}

Figure 1 showed the age distribution of the patients. Maximum $30.3 \%$ patients were belonged to 46 to 60 years age group followed by $26.3 \%$ within $16-30$ years, $17.1 \%$ more than 60 years, $15.8 \%$ within 31 to 45 years and $10.5 \%$ up to 15 years. Mean (SD) age of the patients was $35.95( \pm 18.24)$ and all patients belonged from 4 years to 70 years.

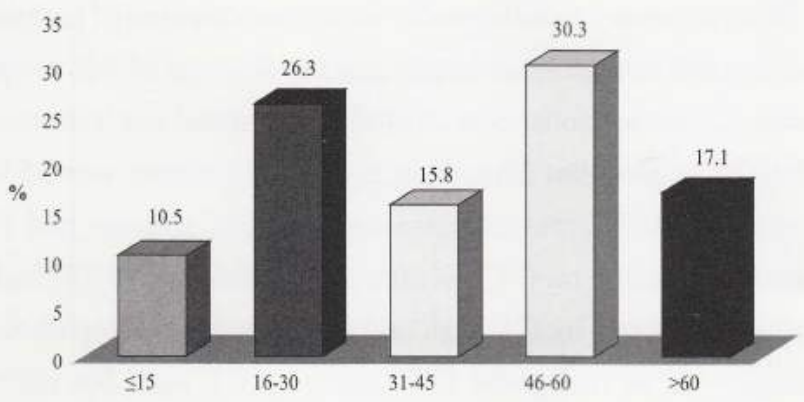

Fig-1: Bar diagram of the patients by age

Table-I

Distribution of patients by clinical presentation $(n=76)$

\begin{tabular}{lcc}
\hline Clinical presentation & Frequency & Percentage \\
Nasal obstruction & 56 & 73.7 \\
Breathing difficulty & 36 & 47.4 \\
Headache & 30 & 39.5 \\
Epistaxis & 16 & 21.1 \\
Eyeache & 9 & 11.8 \\
Proptosis & 8 & 10.5 \\
Gradual swelling of face & 7 & 9.2
\end{tabular}

Table 1 showed the different clinical presentations of patients having PNS mass. Among the patients, 73.7\% had complaints of nasal obstruction, followed by $47.4 \%$ had breathing difficulties, $39.5 \%$ had headache, $21.1 \%$ had epistaxis, $11.8 \%$ had eyeache, $10.5 \%$ had proptosis and $9.2 \%$ had puffiness of their face.

Table-II

Distribution of location of the lesion of PNS $(n=76)$

\begin{tabular}{lcc}
\hline Location of the lesion & Frequency & Percentage \\
\hline Maxillary sinuses & 41 & 53.9 \\
Nasal cavity & 13 & 17.1 \\
Ethmoid sinuses & 11 & 14.5 \\
Sphenoid sinuses & 8 & 10.5 \\
Frontal sinuses & 5 & 6.6 \\
\hline
\end{tabular}


Table II showed the frequency distribution of the PNS mass at different locations. Maximum $53.9 \%$ patients had PNS mass in maxillary sinuses followed by $17.1 \%$ in nasal cavity, $14.5 \%$ in ethmoid sinuses, $10.5 \%$ in sphenoid sinuses and rest $6.6 \%$ in frontal sinuses.

Table-III

$C T$ scan features of PNS masses $(n=76)$

\begin{tabular}{lll}
\hline Frequency & Percentage \\
\hline
\end{tabular}

Margin

- Regular 48

- Irregular 28

Shape

- Rounded 21

- Oval 8

- Irregular 47

Density

- Homogenous 49

- Heterogenous 27

Calcification

3

Fat-fluid level 1

Bone involvement 34

Lymphadenopathy 3

Metastasis

1
Table-IV

Distribution of the masses by CT enhancement pattern

$$
(n=76)
$$

\begin{tabular}{lcc}
\hline & Frequency & Percentage \\
\hline Enhancement $(\mathrm{n}=76)$ & 59 & 77.6 \\
- Present & 17 & 22.4 \\
- Absent & & \\
Enhancement $(\mathrm{n}=59)$ & 10 & 16.9 \\
- Strong enhancement & 6 & 10.2 \\
- Moderate & 43 & 72.9 \\
- Mild & & \\
Pattern of enhancement $(\mathrm{n}=59)$ & & 42.4 \\
- Homogenous & 25 & 57.6 \\
- Heterogenous & 34 & \\
\hline
\end{tabular}

After given contrast $77.6 \%$ masses showed enhancement and of them $10(16.9 \%)$ had strong enhancement, $10.2 \%$ moderate and $72.9 \%$ mild. Among these masses 25 $(42.4 \%$ had homogenous and $34(57.6 \%)$ had heterogenous pattern of enhancement (Table IV).

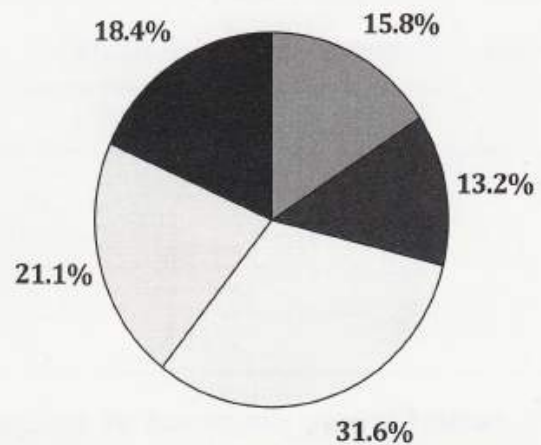

* Multiple responses

Table III showed the different CT scan features of PNS masses. Out of all masses $63.2 \%$ had regular and $36.8 \%$ had irregular margin. $276 \%$ masses were round in shape, $10.5 \%$ oval and $61.8 \%$ irregular. $64.5 \%$ masses had homogenous density and $35.5 \%$ had heterogenous. Out of all mass only $3.9 \%$ had features of calcification, $3.9 \%$ had bony involvement, $3.9 \%$ had lymphadenopathy and $1.3 \%$ had metastatic feature. $44.7 \%$ patients had PNS mass with bony involvement. 


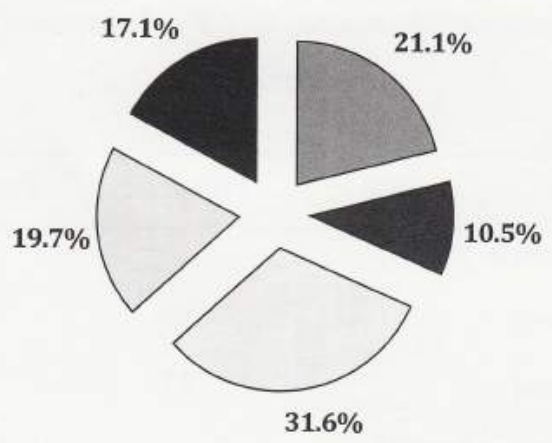

$\square$ Fungal infection $\square$ Inflammatory polyp Benign cyst

Fig-3: Histopathological diagnosis of PNS masses

Figure 3 showed the histopathological diagnosis of PNS masses. Out of 76 cases $31.6 \%$ had inflammatory polyp, $19.7 \%$ had malignant mass, $21.1 \%$ had fungal infection, $10.5 \%$ had nasopharyngeal angiofibroma, and $17.1 \%$ had benign cyst.

Table-V

Distribution of malignant PNS mass by $C T$ scan and histopathological diagnosis

\begin{tabular}{|c|c|c|c|}
\hline \multirow[t]{2}{*}{ CT diagnosis } & \multicolumn{2}{|c|}{ Histopathological diagnosis } & \multirow[t]{2}{*}{ Total } \\
\hline & Malignant & benign & \\
\hline Malignant & 14 & 2 & 16 \\
\hline benign & 1 & 59 & 60 \\
\hline Total & 15 & 61 & 76 \\
\hline
\end{tabular}

Out of all cases 14 were diagnosed as malignant PNS mass by $\mathrm{CT}$ scan and confirmed by histopathological evaluation. They were true positive. Two cases were diagnosed as malignant PNS mass by $\mathrm{CT}$ scan but not confirmed by histopathological findings. They were false positive. Of 60 cases of benign, which were diagnosed by CT scan, one was confirmed as malignant and 59 were benign by histopathology. They were false negative and true negative respectively.
Table-VI

Sensitivity, specificity, accuracy, positive and negative predictive values of the CT in the diagnosis of malignant

\begin{tabular}{lcc}
\multicolumn{3}{c}{ PNS mass } \\
\hline Validity test & Percentage & $95 \%$ CI \\
\hline Sensitivity & 93.3 & $0.766-0.987$ \\
Specificity & 96.7 & $0.926-0.980$ \\
PPV & 87.5 & $0.718-0.925$ \\
NPV & 98.3 & $0.942-0.997$ \\
Accuracy & 96.1 & $0.895-0.982$ \\
\hline
\end{tabular}

Sensitivity of CT scan to diagnose malignant PNS mass was $93.3 \%$, specificity $96.7 \%$, positive predictive value PPV $(87.5 \%)$, negative predictive value NPV (98.3\%) and accuracy $96.1 \%$.

\section{Discussion:}

Mean (SD) age of these patients was $35.95( \pm 18.24)$ and maximum $30.3 \%$ patients were belonged to 46 to 60 years age group. In a previous study done by Rupa et al, similar age group was evaluated. Mean age of their patients was 29.39 years and maximum patients were within 10-19 year age group. ${ }^{8}$ In study by Swamy and Gowda, age incidence for various benign tumours of nose and paranasal sinuses varied from 9 months to 60 years and commonest age group was 11 to 20 years $(36.66 \%)$. Nasal obstruction (73.7\%), breathing difficulties (47.4\%), headache $(39.5 \%)$, epistaxis $(21.1 \%)$, eyeache $(11.8 \%)$, proptosis $(10.5 \%)$ and puffiness face $(9.2 \%)$ were the commonest presenting symptoms of the patients of present study. In study by Swamy and Gowda, nasal obstruction (76.66\%), epistaxis (53\%), and nasal discharge $(50.0 \%)$ were the commonest symptoms followed by mass, headach, hyposmia and nasal twang of speech. ${ }^{9}$ In the present study CT scan evaluated the patients with provisionally diagnosed PNS masses. Out of 76 cases $31.6 \%$ had polyp, $21.1 \%$ had malignant mass, $15.8 \%$ had fungal infection/mass, $13.2 \%$ had nasopharyngeal angiofibroma, and $18.4 \%$ had retention cyst. After radiological diagnosis, all of these patients underwent surgical procedure and histopathological dignosis and 
finally $31.6 \%$ patients were diagnosed as having inflammatory polyp, $19.7 \%$ malignant mass, $21.1 \%$ fungal infection, $10.5 \%$ nasopharyngeal angiofibroma, and $17.1 \%$ benign cyst. In Rupa series out of 33 patients $33.0 \%$ had sinonasal polyp, $9 \%$ fungal infection, $24.24 \%$ angiofibroma, $3 \%$ heamangioma and $27.27 \%$ malignant mass. These findings were evaluated by CT scan. After histopathology $30.3 \%$ patients were diagnosed as polyp, $12.12 \%$ fungal infection, $21.21 \%$ malignant mass, $27.27 \%$ angiofibroma, $6 \%$ haemangioma and $3 \%$ meningioma. $^{8}$

Sensitivity of CT scan to diagnose malignant PNS mass was $93.3 \%$, specificity $96.7 \%$, positive predictive value $87.5 \%$, negative predictive value $98.3 \%$ and accuracy $96.1 \%$. Similar observation was also made by Cagici et al (2005). In their study sensitivity and specificity of threeslice $\mathrm{CT}$ for identifying inflammatory sinus disease were $95.1 \%$ and $92.6 \%$, respectively. ${ }^{10}$ Thirty-two patients with histologically proven malignant disease involving the paranasal sinuses were studied by CT in Parsons and Hodson. ${ }^{11}$ The radiological features of tumor were sinus opacification, a soft-tissue mass, bone erosion and/or displacement, sclerosis, and new-bone formation. Measurements of tissue densities were not helpful in distinguishing malignant tumor from benign disease. Significantly greater tumor extent was demonstrated by CT than by conventional methods in 15 patients; the additional tumor most commonly involved the pterygoid region or orbit. The clinical importance of various directions of tumor spread is emphasized.

\section{Conclusion:}

In conclusion, $\mathrm{CT}$ scan of the malignant para nasal sinus mass provides more information and better image quality and CT diagnosis correlate well with the findings of histopathology. MRI could be added as an additional imaging modality in solvent patients and comparing its diagnostic accuracy with clinical findings CT scan.

\section{References:}

1. Zinreich JS, Kennedy DW, Rosenbaum AE, Gayler BW, Kumar AJ, Stammberger H. Paranasal sinuses: CT imaging requirements for endoscopic surgery. Radiology 1987;163: 769-75.

2. Abayomi OK, Bankoff MS. Computerized tomographic evaluation of tumors of the nasopharynx and maxillary sinus: potential impact on results of treatment. Journal of the National Medical Association 1986;78(4): 311-16.

3. Bhattacharyya N, Fried MP. The accuracy of computed tomography in the diagnosis of chronic rhinosinusitis. Laryngoscope 2003;113(1): 125-29.

4. Reider-Grosswasser I, Solomon A, Zikk D, Godel V. Computerized tomography in conditions concomitantly involving the orbits and the paranasal sinuses. Computerized Radiology 1986;10(2-3): 119-126.

5. Som PM, Shapiro MD, Biller HF, et al. Sinonasal tumors and inflammatory tissues: Differentiation with MR. Radiology 1988;167: 803-808.

6. Duncavage JA, Campbell BH, Hanson Gil. Diagnosis of malignant lymphomas of the nasal cavity, paranasal sinuses and nasopharynx. Laryngoscope 1983;93:1276.

7. Duvoisin B, Landry M, Chapuis L, Krayenbuhl M, Schnyder P. Low-dose CT and inflammatory disease of the paranasal sinuses. Neuroradiology 1991;33( 5): 403-406.

8. Rupa LT, Zimmerman RA. Computed tomography in evaluation of the paranasal sinuses. Radiol Clin North Am 1982; 20: 51-66.

9. Swamy KV, Gowda BVC. A clinical study of benign tumors of nose and paranasal sinuses. Indina Journal of Otolaryngology and Head and Neck Surgery 2004;56(4).

10. Cagici CA, Cakmak O, Hurcan C, Tercan F. Three-slice computerized tomography for the diagnosis and follow-up of rhinosinusitis. European Archives of Oto-Rhino-Laryngology 2005;262(9): 744-50.

11. Parsons C, Hodson N. Computed tomography of paranasal sinus tumors. Radiology 1979;132(3): 641-5. 